\title{
Prevalencia de asma en niños escolares de Mérida, Yucatán
}

\author{
Manuel A. Baeza Bacab y Nelly E. Albertos Alpuche ${ }^{2}$
}

RESUMEN De febrero a junio de 1994 se realizó en la ciudad de Mérida, Yucatán, México, una encuesta para determinar la prevalencia de asma y la frecuencia de antecedentes familiares del padecimiento en niños de edad escolar. Se estudió una muestra aleatoria de 505 niños de 6 a 12 años de edad, de ambos sexos, alumnos de las escuelas de educación primaria del área urbana de Mérida. Se administró una encuesta al padre o a la madre del niño. Se preguntó si el niño había padecido repetidas veces respiración ruidosa con dificultad respiratoria y silbidos, si había padecido bronquitis, bronquitis asmatiforme o asma, y si la madre, el padre o alguno de los hermanos había padecido asma. En total se encontraron 61 niños asmáticos (prevalencia acumulada, 12\%), en los cuales los antecedentes maternos o paternos de asma fueron más frecuentes (25 y 18\%, respectivamente) que en los demás niños (10 y 6\%, respectivamente), siendo la diferencia estadísticamente significativa $(\mathrm{P}<0,002)$ en ambos casos. Los resultados muestran la alta prevalencia de asma en Mérida y sugieren la participación de la herencia en el desarrollo de la enfermedad.

El asma es una de las enfermedades crónicas infantiles más frecuentes y constituye una causa importante de ausentismo escolar y limitación funcional (1). En la última década se ha observado un incremento de su prevalencia en diversos países (2-4). Los estudios epidemiológicos en América Latina han revelado prevalencias variables de 5,7 a 16,5\% (5-7) que pueden deberse a las distintas metodolo-

\footnotetext{
1 Universidad Autónoma de Yucatán, Facultad de Medicina, Mérida, Yucatán, México. La correspondencia ha de enviarse a este autor a la siguiente dirección postal: Facultad de Medicina, Universidad Autónoma de Yucatán, Avenida Itzaés, No. 498 por calle 59-A, C.P. 97.000 Mérida, Yucatán, México. Correo electrónico: bbacab@tunku.uady. $\mathrm{mx}$

2 Universidad Autónoma de Yucatán, Centro de Investigaciones Regionales “Dr. Hideyo Noguchi", Mérida, Yucatán, México.
}

gías empleadas en los estudios o a la influencia de diversos factores de riesgo. Los resultados de estas investigaciones no siempre pueden extrapolarse a otras regiones, lo que hace conveniente determinar las características epidemiológicas del asma en cada región.

En México no se ha estimado la prevalencia de asma en la población general del país o en grupos definidos de edad y sexo. Sin embargo, se han publicado estimaciones de la prevalencia de asma en varias zonas del país. En Villahermosa, Tabasco, una encuesta realizada en 1986 mostró una prevalencia acumulada de $12,5 \%$ en la población escolar (8). En Guadalajara, Jalisco, en 1990, se halló una prevalencia en la población general de $13 \%$, con predominio de la enfermedad en el sexo masculino (9). En el estado de Yucatán no se había estimado hasta ahora la prevalencia del asma en niños, pero se había observado que los episodios agudos del padecimiento ocupan un lugar importante como causa de consulta en el servicio de urgencias del Hospital General O'Horán de la Secretaría de Salud en la ciudad de Mérida (10). Por ello decidimos realizar una encuesta en niños escolares de la ciudad de Mérida, capital del estado de Yucatán, para determinar la prevalencia acumulada de asma - es decir, la proporción de individuos que han padecido asma en algún momento de su vida, también llamada "prevalencia de vida" (11) así como la relación del asma con los antecedentes familiares del mismo padecimiento. 


\section{MATERIALES Y MÉTODOS}

La población de estudio se compuso de niños o niñas de 6 a 12 años de edad que acudían a las escuelas de educación primaria localizadas en la zona urbana de Mérida. Según la Dirección de Educación Primaria de la Secretaría de Educación Pública del Estado de Yucatán, dicha población consta de 82033 escolares distribuidos en 200 escuelas primarias y se estima que $5 \%$ de la población infantil entre las edades de 6 y 12 años no se encuentra escolarizada.

Se seleccionó una muestra aleatoria mediante muestreo probabilístico multietápico. El tamaño muestral se calculó previamente mediante el programa Epistat, suponiendo una prevalencia de $15 \%$, con un margen admisible de error absoluto de $5 \%$ y un nivel de confianza de $99 \%$. Con dichas especificaciones el tamaño muestral mínimo es 337. Dicho tamaño se incrementó hasta 505 para conseguir mayor precisión de la estimación. El número de alumnos que había de seleccionarse en cada escuela se calculó como parte proporcional de la muestra a partir de la proporción que representaba esa escuela en la población de estudio. Finalmente se seleccionaron los alumnos participantes mediante muestreo aleatorio simple, independiente de su edad y sexo, utilizando los registros de las escuelas.

El cuestionario que se usó (cuadro 1) se elaboró a partir de las preguntas de otras encuestas realizadas en el país $(8,9)$. Con este cuestionario se llevó a cabo una prueba piloto del 1 de mayo al 30 de junio de 1993 en una escuela primaria de la ciudad de Mérida. Los encuestadores fueron estudiantes de la Facultad de Medicina de la Universidad Autónoma de Yucatán, a quienes se instruyó sobre cómo aplicar el cuestionario, que fue luego administrado por esos estudiantes al padre o la madre de 180 alumnos. Se evaluaron la idoneidad y la claridad de cada pregunta y las que resultaron problemáticas se reformularon.

Del 15 de abril al 30 de junio de 1994 se administraron las encuestas a los

CUADRO 1. Cuestionario utilizado en una encuesta sobre prevalencia del asma en escolares de Mérida, Yucatán

No.
Nombre del niño _
Edad__ Fecha__
1) ¿Su hijo ha padecido respiración ruidosa con dificultad respiratoria y silbidos
en forma repetida?
2) ¿Su hijo ha padecido bronquitis?
¿El diagnóstico fue hecho por un médico?
3) ¿Su hijo ha padecido bronquitis asmatiforme?
¿El diagnóstico fue hecho por un médico?
4) ¿Su hijo ha padecido asma?
5) ¿La madre padece o ha padecido asma?
6) ¿El padre padece o ha padecido asma?
7) ¿Alguno de los hermanos padece o ha padecido asma?

adultos responsables de los escolares que constituyeron la muestra.

Los cuestionarios definitivos fueron entregados en las escuelas al padre o a la madre de cada niño seleccionado y fueron cumplimentados directamente por el adulto responsable bajo la supervisión de uno de los encuestadores. No hubo rechazos a participar.

Se consideró asmático al niño con dos respuestas afirmativas a " ¿Su hijo ha padecido asma?" y “ ¿El diagnóstico fue hecho por un médico?" (pregunta 4). La prevalencia acumulada fue el porcentaje de niños asmáticos en el total de la muestra o en los subgrupos correspondientes.

Se empleó la prueba de ji cuadrado $\left(\chi^{2}\right)$ para estimar la intensidad de la asociación estadística entre el asma en el niño y el antecedente de la enfermedad en uno o ambos progenitores. Para los análisis estadísticos se utilizó el programa Epi Info.

\section{RESULTADOS}

La muestra se compuso de un total de 505 escolares (248 niñas y 257 niños) y se distribuyó más o menos homogéneamente entre las distintas edades, predominando entre las niñas las de 9 años (43 niñas) y entre los niños los de 10 años (también 43). La edad menos representada en la muestra fueron los 12 años en el sexo femenino (26 niñas) y los 6 años en el masculino (28 niños).

Se detectaron 61 "asmáticos" según la definición antes indicada, lo que corresponde a una prevalencia acumulada de $12 \%$. El grupo de asmáticos estuvo formado por 33 niños y 28 niñas. Por edades, los asmáticos predominaron entre las niñas a los 7 y 8 años de edad y entre los niños a los 10, 11 y 12 años. Las diferencias no fueron estadísticamente significativas.

En el grupo de niños asmáticos se encontraron antecedentes maternos o paternos de la enfermedad en $25 \%$ (15) y $18 \%$ (11) de los cuestionarios, respectivamente. Entre los escolares no asmáticos solo hubo antecedentes asmáticos maternos en $10 \%$ (46) y paternos en $6 \%$ (28). La asociación fue estadísticamente significativa, tanto para el antecedente asmático materno $\left(\chi^{2}=\right.$ $10,2$, g.l. $=1, P<0,002)$ como para el antecedente paterno $\left(\chi^{2}=10,3, P<\right.$ $0,002)$. Los antecedentes familiares de asma considerados en conjunto (padre, madre o hermanos con asma) 
fueron positivos en $30 \%(152 / 505)$ de la muestra estudiada, en 52\% (32/61) del grupo de asmáticos y en 27\% (120/ 444) del grupo de no asmáticos. La razón de frecuencias de antecedente asmático familiar es $1,9(=52 / 27)$, lo que significa que en el grupo que había padecido asma la frecuencia de antecedentes familiares de la enfermedad fue casi el doble en comparación con la de los no asmáticos.

\section{DISCUSIÓN}

Las encuestas constituyen el método más fácil para realizar estudios epidemiológicos en los que se identifican individuos asmáticos (12), aunque sus resultados pueden no ser tan exactos como los que se obtienen al utilizar pruebas de broncoprovocación para el diagnóstico de la enfermedad. Cuando se utiliza una encuesta, es posible que otros padecimientos que cursan con sibilancias interfieran con los resultados, que los individuos encuestados no tengan la capacidad suficiente para proporcionar los datos apropiados, o que las respuestas sean tan variadas que no permitan su agrupamiento para su análisis posterior. Por todo ello, en nuestro trabajo solo fueron entrevistados los adultos responsables y solo se consideraron asmáticos los niños previamente diagnosticados por un médico. Según los resultados obtenidos, la prevalencia de asma en los niños escolares del área urbana de Mérida, $12 \%$, es similar a la alta prevalencia encontrada en otras encuestas, tanto en México como en otros países $(3,5,8,9)$.

A pesar de que nuestro trabajo no tuvo como objetivo explicar las causas de la enfermedad, creemos que la alta prevalencia encontrada puede estar relacionada con factores hereditarios, ya que los antecedentes familiares de asma fueron mucho más frecuentes en los escolares asmáticos. En diversas publicaciones $(13,14)$ se han notificado antecedentes familiares en los niños asmáticos y se ha comprobado (15) que los genes que gobiernan la hiperreactividad bronquial están localizados en el brazo largo del cromosoma 5. Además, en los últimos años diversos países han notificado un incremento del número de casos de asma diagnosticados cuya razón todavía no se ha determinado, pero se considera que el incremento ha sido demasiado rápido para deberse a factores genéticos. Más bien parece asociarse con factores ambientales, tales como un mayor número de alergenos, el tabaquismo en mujeres de edad reproductiva, la dieta, la contaminación del aire dentro y fuera de la casa y las infecciones de origen viral (16). Recientemente se demostró que los niños asmáticos con alergia a las cucarachas tienen un mayor número de consultas, hospitalizaciones, ausencias escolares y trastornos del sueño como consecuencia de su padecimiento (17).

Los resultados del estudio sugieren que el asma constituye un problema de salud pública en la región, ya que un porcentaje importante de los escolares $(12 \%)$ padecen de esta enfermedad crónica, que se puede acompañar de una morbilidad elevada y de limitaciones funcionales. También se observó que 30\% de las familias encuestadas tenían, cuando menos, un miembro con dicho padecimiento.

Agradecimiento. Los autores agradecen su amable colaboración en la aplicación de las encuestas a Daniel I. Sánchez, Miroslava Segovia, Claribel Segura, Rosselud Solano, Norma Tuyú, Ricardo Vallado, Alberto Ventura y Luis Villegas, todos ellos estudiantes de la Facultad de Medicina de la Universidad Autónoma de Yucatán.

\section{REFERENCIAS}

1. Carter-Pokras OD, Gergen PJ. Reported asthma among Puerto Rican, Mexican-American, and Cuban children, 1982 through 1984. Am J Public Health 1993;83:580-582.

2. Wood PR, Hidalgo HA, Prihoda TJ, Kromer ME. Hispanic children with asthma: morbidity. Pediatrics 1993;91:62-69.

3. Crain EF, Weiss KB, Bijur PE, Hersh M, Westbrook L, Stein REK. An estimate of the prevalence of asthma and wheezing among inner-city children. Pediatrics 1994;94: 356-362.

4. Robertson CF, Heycock E, Bishop J, Nolan T, Olinsky A, Phelan PD. Prevalence of asthma in Melbourne schoolchildren: changes over 26 years. Brit Med J 1991;302:1116-1118.

5. Caraballo L, Cadavid A, Mendoza J. Prevalence of asthma in a tropical city of Colombia. Ann Allergy 1992;68:525-529.

6. Fritscher CC, Severo RD, Fagondes SC, Cohen R, Dornelles RL, Kahan F. Modificações na prevalência de asma bronquica em escolares de Porto Alegre. J Pneumol 1994;20:6-10.

7. Cruz Bournigal E, Márquez Rodríguez R, Ortiz González M, Acevedo Acosta G. Prevalencia del asma bronquial en escolares de 6-14 años en Santo Domingo, República Dominicana. Arch Dom Pediatr 1985;21:9-14.
8. Baeza Bacab MA, Graham Zapata LF. Prevalencia del asma: encuesta en una población escolar de Villahermosa, Tabasco, México. Alergia 1992;39:32-36.

9. González Gómez JG, Barrera Gómez LE, Arévalo Orozco MA. Prevalencia del asma bronquial en la ciudad de Guadalajara, Jalisco, México. Alergia 1992;39:3-7.

10. Pacheco Medina FA, Baeza Bacab MA. Asma y clima: relación entre cambios climatológicos y asma aguda en niños que acudieron al servicio de Urgencias Pediátricas del Hospital General O'Horán del 1 de enero al 31 de diciembre de 1990. Cuadernos de la Facultad de 
Medicina, Universidad Autónoma de Yucatán 1993;15:135-136.

11. Greeg I. Epidemiological aspects. En: Clark TJH, Godfrey S, eds. Asthma. London: Chapman and Hall Medical; 1983:242-284.

12. Samet JM. Epidemiologic approaches for the identification of asthma. Chest 1987;91: 74S-78S.

13. Peat JK, Brittom WJ, Salome CM, Woolcock AJ. Bronchial hyperresponsiveness in two populations of Australian schoolchildren: IIRelative importance of associated factors. Clin Allergy 1987:17;283-290.
14. Fergusson DM, Horwood LJ, Shannon FT. Parental asthma, parental eczema and asthma and eczema in early childhood. J Chronic Dis 1983;36:517-524.

15. Postma DS, Bleecker ER, Amelung PJ, Holroyd KJ, Xu J, Panhuysen CIM, et al. Genetic susceptibility to asthma-bronchial hyperresponsiveness coinherited with a major gene for atopy. N Engl J Med 1995;333:894-900.

16. Newman-Taylor A. Environmental determinants of asthma. Lancet 1995;345:296-299.

17. Rosenstreich DL, Eggleston P, Kattan M, Baker D, Slavin RG, Gergen P, et al. The role of cockroach allergy and exposure to cockroach allergen in causing morbidity among inner-city children with asthma. $N$ Engl J Med 1997;336:1356-1363.

Manuscrito recibido el 9 de julio de 1996 y aceptado para publicación, tras revisión, el 20 de noviembre de 1996.

ABSTRACT A survey was conducted in Merida, Yucatan, between February and June 1994 in order to determine the prevalence of asthma and the frequency of a family history of the disease in school-age children. A random sample was taken of 505 children of both sexes between the ages of 6 and 12 who were enrolled in elementary schools in the urban area of Merida. Every child's father or mother was surveyed and asked if the child had had recurrent episodes of loud, difficult breathing with wheezing chest sounds, if he or she had had bronchitis, asthmatiform bronchitis, or asthma, and if the mother, father, or any of the siblings had ever suffered from asthma. A total of 61 children with asthma (cumulative prevalence, 12\%) was found. Among these children a history of maternal or paternal asthma was more frequent ( 25 and $18 \%$, respectively) than in other children (10 and $6 \%$, respectively), the difference being statistically significant $(P<0,002)$ in both cases. Results show the high prevalence of asthma in Merida and suggest that heredity plays a role in the development of the disease. 